\title{
Extensive Metastatic Non-Small Cell Lung Adenocarcinoma in Young Age Group
}

\author{
Hassan Awada* and Yihong Zhou \\ Department of Internal Medicine, Cleveland Clinic, Cleveland, Ohio, USA \\ *Corresponding author: Hassan Awada, Department of Internal Medicine, Cleveland Clinic, Cleveland, Ohio USA
}

\section{ARTICLE INFO}

Received: 蔧 June 11, 2019

Published: 幽 June 18, 2019

Citation: Hassan Awada, Yihong Zhou. Extensive Metastatic Non-Small Cell Lung Adenocarcinoma in Young Age Group. Biomed J Sci \& Tech Res 18(5)2019. BJSTR. MS.ID.003221.

\section{ABSTRACT}

Adenocarcinoma of the lung is a subtype of non-small cell lung cancer which contributes to $40 \%$ of all lung cancers. It is mainly seen in older population and very rarely diagnosed in young patients. Here we report a very rare and uncommon presentation of stage IV lung adenocarcinoma in a 26-year-old female with brain, liver and bone metastases.

\section{Introduction}

Lung cancer is the leading cause of cancer death in both men and wen. It is divided into three main categories that include non-small cell lung cancer (NSCLC; 80-85\%), small cell lung cancer (SCLC; 10$15 \%)$ and lung carcinoid tumor (5\%). It mainly occurs in elderly Case age group (65 years or older) with an average age of 70 years at the time of diagnosis. However, it is very rare in young population with no identified risk factors, environmental exposures, or genetic predisposition.

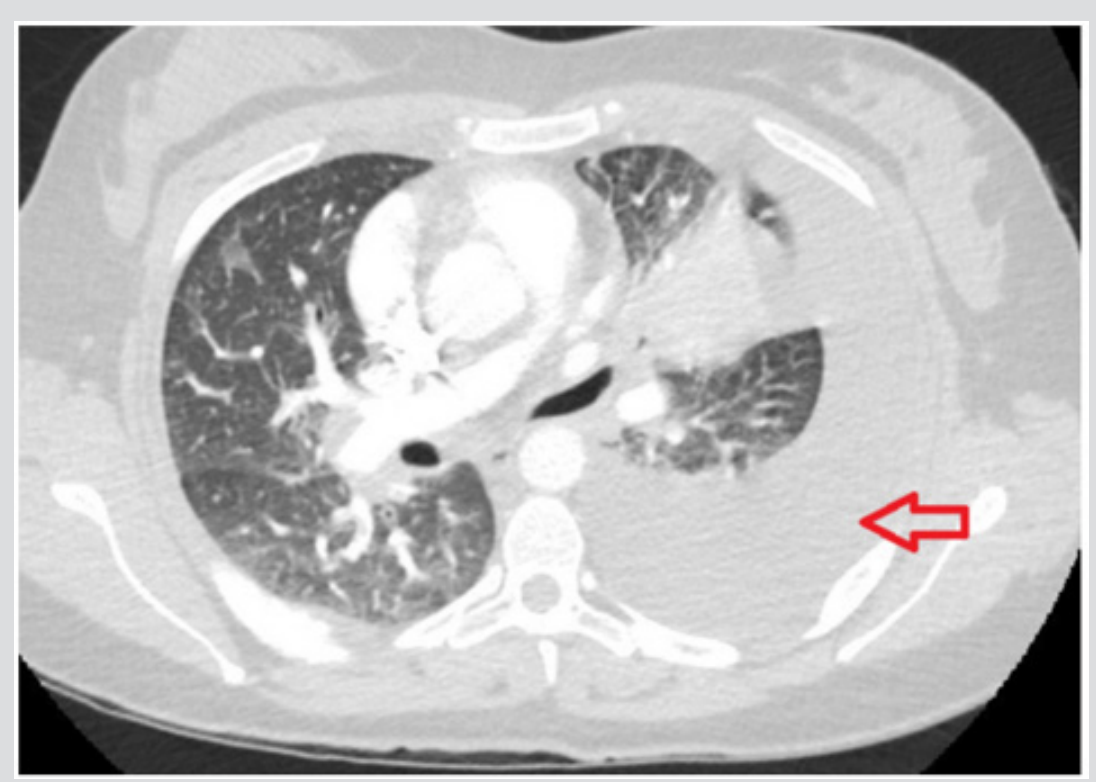

Figure 1: A computed tomography imaging of the chest with intravenous contrast showing very large left-sided pleural effusion and soft tissue density extending outward from the left hilum with the possibility of an underlying mass. 
A 26-year-old female with insignificant past medical history presented to the emergency department with worsening shortness of breath following an upper respiratory tract infection for which she completed a course of azithromycin (Z-pack). A chest x-ray was done which showed abnormal parenchymal opacity at the left lower lung consistent with atelectasis and/or infiltrate, in addition to a small left effusion. A computed tomography imaging (CT scan) of the chest with intravenous (IV) contrast ruled out pulmonary embolism but showed very large left-sided pleural effusion and soft tissue density extending outward from the left hilum representing atelectasis with the possibility of an underlying mass (Figure 1). The patient had thoracentesis of her left lung and the pathological analysis of the pleural fluid showed exudates with malignant cells. Trans-abdominal ultrasound (US) to rule out Meigs syndrome showed no acute findings, however, it did show a heterogeneous lesion measuring up to $1.5 \mathrm{~cm}$ within the musculature of the right lower abdominal wall which corresponded to the palpable abnormality on her physical examination. US ascites survey showed no abdominal ascites. US pelvis showed $1.5 \mathrm{~cm}$ intramuscular lesion. CT of the abdomen and pelvis showed no acute process but a subtle $1.8 \mathrm{~cm}$ hypodense lesion in the right hepatic lobe that is indeterminant. Flexible bronchoscopy with bronchoalveolar lavage and biopsies was performed.

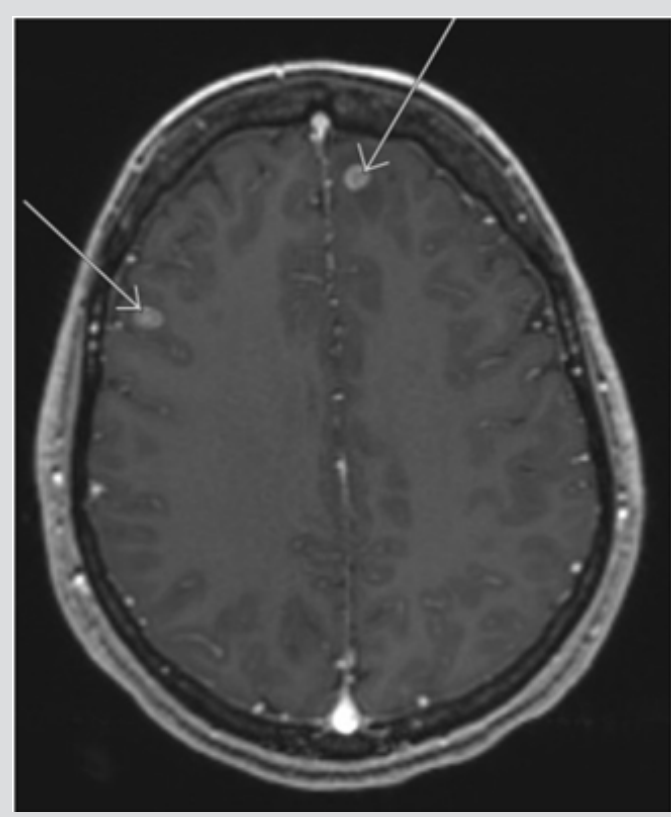

Figure 2: Magnetic resonance imaging of the brain showing multiple scattered enhancing masses situated along the gray-white interface throughout both cerebral hemispheres compatible with metastases.

It showed evidence of stage IV adenocarcinoma with immunohistochemistry positive for anaplastic lymphoma kinase (ALK-D5F3) in the neoplastic cells. Oncology service was notified, and the patient was scheduled for outpatient chemotherapy. A follow up magnetic resonance imaging of the brain (MRI) showed multiple scattered enhancing masses situated along the gray-white interface throughout both cerebral hemispheres compatible with metastases (Figure 2). Nuclear medicine positron emission tomography (PET)/ CT scan of the skull to thigh showed multiple hypermetabolic areas suspicious for neoplastic process and metastases including the right supraclavicular lymph node, left perihilar region, right posterior lateral chest wall/rib cage, right anterior chest wall/subpectoral region, right lower anterior abdominal wall and right medial thigh. Moreover, it showed hypermetabolic liver lesion concerning for liver metastases and scattered skeletal hypermetabolic lesions suspicious for osseous metastasis including lytic sclerotic lesion in the left proximal femur (Figure 3). Taking into account her ALK positive status, the patient was planned for therapy with Alectinib and was scheduled for pleurx catheter placement by thoracic surgery to manage her pleural effusions.

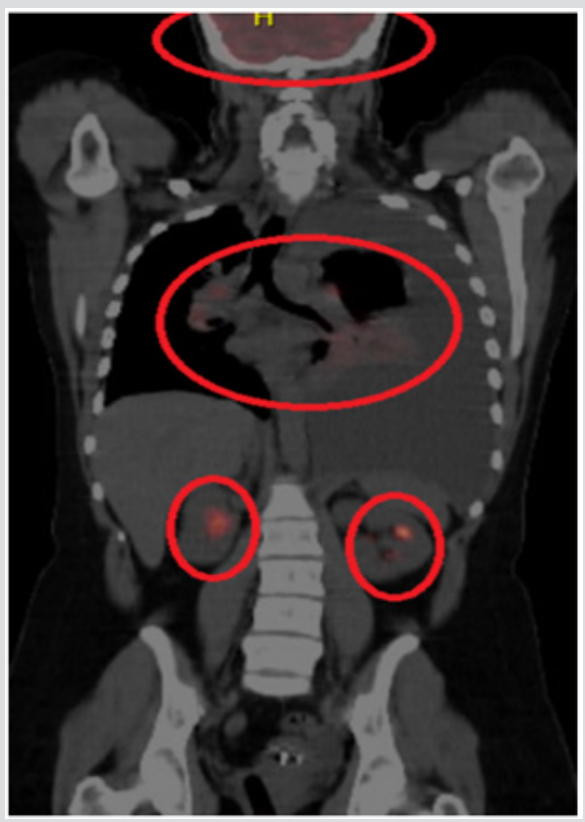

Figure 3: Nuclear medicine positron emission tomography of the skull to thigh showing multiple hypermetabolic areas suspicious for neoplastic process and metastases.

\section{Discussion}

Among all types of lung cancers, NSCLC is the most common [1]; and of NSCLC subtypes, adenocarcinoma is the highest in prevalence. Although tobacco smoking is the strongest risk factor for lung cancers, it is less likely associated with adenocarcinoma as compared to SCLC and squamous cell carcinoma [2]. Age has been recognized as a risk factor for all lung cancers. In young population, NSCL along with all other lung cancers are extremely rare with an incidence of $0.3-0.5 \%$ [3-4]. Interestingly, among this minor population, adenocarcinoma represents the highest portion (48\%). In this patient, who has not had any modified risk for lung cancer, environmental exposures, or genetic predisposition, extensive metastasis to the brain, liver, abdominal wall and bone was very surprisingly seen at time of diagnosis. This, in fact, reflects the aggressiveness of lung adenocarcinoma which tend to be detected at advanced stages when diagnosed in young adults. 


\section{Conclusion}

Our case report highlights the rare incidence of lung adenocarcinoma with extensive metastases in young adults. It adds to the literature an uncommon presentation that questions the need for future studies and clinical trials for evaluation of metastatic lung cancer in young age groups.

\section{References}

1. Jemal A, Bray F, Center MM, Ferlay J, Ward E, et al. (2011) Global cancer statistics. CA Cancer J Clin 61(2): 69-90.

\section{ISSN: 2574-1241}

DOI: 10.26717/BJSTR.2019.18.003221

Hassan Awada. Biomed J Sci \& Tech Res

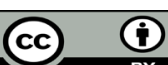

This work is licensed under Creative Commons Attribution 4.0 License

Submission Link: https://biomedres.us/submit-manuscript.php
2. Molina JR, Yang P, Cassivi SD, Schild SE, Adjei AA, et al. (2008) Non-small cell lung cancer: epidemiology, risk factors, treatment and survivorship. Mayo Clin Proc 83(5):584-594

3. Rich AL, Khakwani A, Free CM, Tata LJ, Stanley RA, et al. (2015) Nonsmall cell lung cancer in young adults: presentation and survival in the English National Lung Cancer Audit. QJM 108(11): 891-897.

4. Bethesda (2009) United States, National Institutes of Health, National Cancer Institute, Surveillance Epidemiology and End Results (seer) Cancer Statistics Review, 1975-2006, National Cancer Institute, USA, Cancer Statistics Branch.

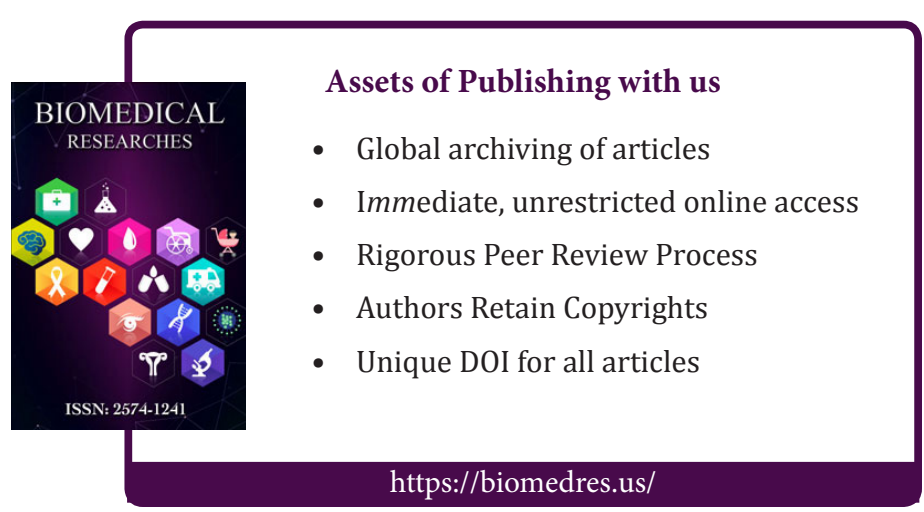

